MATHEMATICS OF COMPUTATION

S 0025-5718(99)01075-3

Article electronically published on February 19, 1999

\title{
A FINITE ELEMENT APPROXIMATION FOR A CLASS OF DEGENERATE ELLIPTIC EQUATIONS
}

\author{
BRUNO FRANCHI AND MARIA CARLA TESI
}

\begin{abstract}
In this paper we exhibit a finite element method fitting a suitable geometry naturally associated with a class of degenerate elliptic equations (usually called Grushin type equations) in a plane region, and we discuss the related error estimates.
\end{abstract}

\section{INTRODUCTION}

Let $\Omega$ denote the bounded subset of $\mathbb{R}^{2}=\mathbb{R}_{x} \times \mathbb{R}_{y}$ defined by $\left.\Omega=\right]-1,1[\times]-1,1[$, and let $\Gamma$ be its boundary. We consider the second order differential operator in divergence form in $\Omega$ defined by

$$
\mathcal{L}=-\sum_{i, j=1}^{2} \partial_{i}\left(a_{i j}(z) \partial_{j}\right),
$$

where the coefficients $a_{i j}=a_{j i}$ are measurable real-valued functions and, for some $\nu \in(0,1)$,

$$
\nu\left(\xi^{2}+\lambda^{2}(x) \eta^{2}\right) \leq \sum_{i, j=1}^{2} a_{i j}(z) \zeta_{i} \zeta_{j} \leq \frac{1}{\nu}\left(\xi^{2}+\lambda^{2}(x) \eta^{2}\right)
$$

for any $\zeta=\left(\zeta_{1}, \zeta_{2}\right)=(\xi, \eta)$ and $z=(x, y) \in \mathbb{R}^{2}$. Here $\lambda$ is a bounded nonnegative Lipschitz continuous function in $\mathbb{R}$. For simplicity, the reader can think of a model operator of the form

$$
\mathcal{L}_{0}=-\partial_{1}^{2}-\lambda^{2}(x) \partial_{2}^{2} .
$$

Operators of this form are known as Grushin type operators, and regularity properties of the weak solutions of $\mathcal{L} u=f$ have been widely studied in the last few years: see, for instance, $[\mathrm{FL}],[\mathrm{X}],[\mathrm{FS}],[\mathrm{F}],[\mathrm{FGuW} 1],[\mathrm{FGuW} 2]$. Grushin operators can be viewed as (generalized) Tricomi operators for transonic fluids restricted to the subsonic region. In addition, note that every second order differential operator in divergence form on the plane with nonnegative principal part and which is not totally degenerate at any point (i.e. its quadratic form does not vanish identically at any point) can be written, after a suitable change of variables, as an operator whose principal part is a Grushin type operator (see $[\mathrm{X}]$ for an explicit calculation).

Received by the editor June 28, 1996 and, in revised form, September 8, 1997 and March 31, 1998.

1991 Mathematics Subject Classification. Primary 46E30, 49N60.

The first author is partially supported by M.U.R.S.T., Italy (40\%) and by G.N.A.F.A. of C.N.R., Italy (60\%).

The authors are indebted to A. Valli for many fruitful discussions. 
A fruitful approach to the study of these operators was shown (see [FL]) to consist in associating with the operator $\mathcal{L}$ a suitable (non-Riemannian) metric $d$ which is basically given by the minimum time required to pass from a given point to another along continuous curves which are piecewise integral curves of the vector fields $\pm \partial_{1}$ and $\pm \lambda \partial_{2}$ (see Definition 2.1 for a precise definition). If for instance we are interested in the Hölder continuity of the weak solutions (De Giorgi-Nash-Moser theorem) or in Harnack's inequality for positive weak solutions, then we can repeat the classical arguments developed for elliptic equations ([DG], $[\mathrm{Mo}]$ ) by replacing the usual Euclidean balls by the so-called metric balls, i.e. by the balls of the metric $d$.

The aim of the present paper is to show that a similar geometric approach can lead to a natural finite element method for this class of operators. In fact, we shall exhibit a triangulation of a plane region by means of a family of nonisotropic triangles fitting the geometry associated with the metric $d$, in the sense that each triangle of our triangulation contains and is contained in two metric balls of comparable radii. The shape of these triangles will not be trivial to describe, since metric balls are not invariant under Euclidean translations, so that we cannot just repeat a fixed ball by translation. Analogously, there are no simple dilations enabling us to rescale our geometry or our estimates.

In a similar spirit, a finite difference method for ultraparabolic equations of Kolmogorov type has recently been developed in [MP].

We point out that our approach is not precisely an adaptive method, since, roughly speaking, the geometry is fixed a priori and it is given by our model operator $\partial_{1}^{2}+\lambda^{2}(x) \partial_{2}^{2}$, which plays the role of a Laplace-Beltrami operator for our geometry. An adaptive method might be superposed on this choice of the geometry, keeping in mind the oscillation of the coefficients (note that, in this spirit, the function $\lambda$ is not a coefficient, but a structure term).

We note explicitly that, because of the lack of ellipticity when $\lambda$ vanishes, we are forced to seek weak solutions belonging to function spaces which are larger than the usual Sobolev space $\stackrel{\circ}{H}^{1}(\Omega)$ and that are given by the completion of $C_{0}^{\infty}(\Omega)$ with respect to the norm

$$
\|u\|_{L^{2}(\Omega)}+\left\|\partial_{1} u\right\|_{L^{2}(\Omega)}+\left\|\lambda \partial_{2} u\right\|_{L^{2}(\Omega)},
$$

so that in general our weak solutions do not belong to $\stackrel{\circ}{H}^{1}(\Omega)$.

In fact, this approach has been used for a much larger class of degenerate elliptic operators, whose prototype is given by Hörmander's well known sum-of-squares operators in $\mathbb{R}^{n}$ of the form $\sum_{j=1}^{n} X_{j}^{2}$, where $X_{1}, \ldots, X_{n}$ are smooth vector fields such that the rank of the Lie algebra generated by them equals $n$ at any point. For instance, if we choose $\lambda(x)=|x|^{k}$, for some positive integer $k$, then our model operator $\partial_{1}^{2}+|x|^{2 k} \partial_{2}^{2}$ is a Hörmander operator. Since we are dealing with nonsmooth functions $\lambda$, we shall have to impose further conditions on $\lambda$ to replace this rank hypothesis (see Hypothesis $(\mathrm{H})$ below).

If we try to follow the scheme of Moser's proof of the pointwise regularity of the weak solutions of $\mathcal{L} u=f$, two points appear from the beginning to play a crucial role: the fact that the metric $d$ is doubling (i.e. the volume of a metric ball of radius $2 r$ is controlled by a constant times the volume of a ball of radius $r$ having the same center), and a suitable Sobolev-Poincaré inequality on metric balls, where, on the right hand side, we have to replace the usual gradient $\nabla u$ by the 'degenerate 
gradient' $\nabla_{\lambda} u=\left(\partial_{1}, \lambda \partial_{2}\right)$ associated with the operator. These inequalities contain deep information concerning the geometry associated with the metric $d$, since they show that the geometric dimension of the metric space defined by $d$ is much larger than 2 (or than $n$ in general) and, roughly speaking, it is as large as $\lambda$ is degenerate. This phenomenon has been studied in the general context of Hörmander's vector fields, and it appears clearly in a family of isoperimetric inequalities associated with a family of such vector fields (see [FGaW], [FLW], [CDG1] [CDG2], [GN], [Gr]).

Unfortunately, this dimensional phenomenon affects our error estimates negatively. Indeed, first of all, we do not have any Sobolev imbedding theorem to control the pointwise values of a weak solution in the interpolation operator by means of some higher Sobolev norm, as in the elliptic case. Roughly speaking, this estimate is possible for a function $u \in H^{s}\left(\mathbb{R}^{n}\right)$ if $n<2 s$, and, as we pointed out before, the dimension of $(\Omega, d)$ is in general much higher than 2 . Nevertheless, it is possible to bypass this difficulty, but the same dimensional phenomenon appears again in the numerical approximation, since, corresponding to a mesh of $N$ points, we find in the error estimate a factor $N^{-1 /(2 \gamma+2)}$, where $\gamma \geq 0$ and $\gamma+2$ is basically the geometric dimension of $(\Omega, d)$ (all these quantities will be defined formally later). In other words, a large number of triangles is required to obtain small errors, much larger than in the elliptic case, and larger and larger as $\lambda$ becomes 'flat' at the points where it vanishes, so that our approximation converges, but the rate of convergence is affected by the order of degeneration of the function $\lambda$. Then, it is necessary to take this phenomenon into account when we compare our numerical results with those we can obtain just by running numerical elliptic procedures outside of any theoretical scheme. Indeed, this naïve approach gives locally good results away from the zeros of $\lambda$ (since the operator $\mathcal{L}$ is locally elliptic in these regions). Note that, as we shall discuss later by means of numerical examples, our error estimates are sharp.

In Section 2 we characterize the geometry associated to a given class of operators, in Section 3 we set up the general framework for a finite element method fitting the given geometry, in Section 4 we prove error estimates and in Section 5 we discuss the algorithmic implementation of the method, and we show, by means of a suitable choice of the right hand side of the equation, that - as we can expect - the error estimate in the energy norm can be better than the error we obtain by using a standard mesh, or even an adaptive one (but we stress again that the use of a standard mesh has no justification, since there are solutions which do not belong to the usual Sobolev space $H^{1}(\Omega)$ ). In addition, we exhibit numerical examples showing that our error estimate is optimal. This will be done by analyzing the error (in the energy norm associated with the operator) when the data have been chosen in such a way that the solution does not belong to the usual Sobolev space.

\section{Preliminaries}

Through this paper we will denote a generic point in $\mathbb{R}^{2}$ by $z=(x, y)$. In the sequel, we will assume that the function $\lambda$ satisfies the following assumption:

Hypothesis $(\mathbf{H})$. There exists a positive constant $c_{1}$ such that, for any compact interval $I \subseteq \mathbb{R}$,

$$
0<c_{1} \max _{I} \lambda \leq \frac{1}{|I|} \int_{I} \lambda(x) d x \leq \max _{I} \lambda,
$$

where $|I|$ denotes the Lebesgue measure of $I$. 
This condition is called the $R H_{\infty}$ condition in [F] and [FGuW1], and it implies basically that $\lambda$ is not flat where it vanishes. For instance, if $p$ is any polynomial in $x_{1}$, then $\lambda\left(x_{1}\right)=\left|p\left(x_{1}\right)\right|^{\alpha}(\alpha \geq 1)$ belongs to $R H_{\infty}$. Indeed, by rescaling, we can reduce ourselves to proving that $\max _{[0,1]}|q|^{\alpha} \approx \int_{0}^{1}|q(x)|^{\alpha} d x$ when $q$ is a polynomial of degree $\leq m, m$ fixed. But both $\max _{[0,1]}|q|$ and $\left(\int_{0}^{1}|q(x)|^{\alpha} d x\right)^{1 / \alpha}$ are norms on the finite-dimensional linear space of all polynomials of degree $\leq m$, and so they are equivalent. For some comments concerning the intrinsic geometric meaning of $R H_{\infty}$, see also [CF].

Let us recall now the definition of the metric associated with a family of vector fields $\left\{\lambda_{1} \partial_{1}, \ldots, \lambda_{n} \partial_{n}\right\}$ (see $[\mathrm{FP}],[\mathrm{FL}],[\mathrm{NSW}]$ ) and the main results we will need through this paper.

The distance we shall define is sometimes called Carnot-Carathéodory distance, or control distance: indeed, it arises naturally in many optimal control probles (see, e.g., recent accounts in $[\mathrm{J}]$ ).

Definition 2.1. We say that an absolutely continuous curve $\gamma:[0, T] \rightarrow \mathbb{R}^{2}$ is a sub-unit curve if for any $\zeta=(\xi, \eta) \in \mathbb{R}^{2}$,

$$
\langle\dot{\gamma}(t), \zeta\rangle^{2} \leq|\xi|^{2}+\lambda^{2}(\gamma(t))|\eta|^{2}
$$

for a.e., $t \in[0, T]$ (note that to simplify our notation we have considered $\lambda$ here as a function of $z \in \mathbb{R}^{2}$ ). If $z_{1}, z_{2} \in \mathbb{R}^{2}$, we put

$$
\begin{gathered}
d\left(z_{1}, z_{2}\right)=\inf \left\{T>0 ; \text { there exists a sub-unit curve } \gamma:[0, T] \rightarrow \mathbb{R}^{2}\right. \\
\text { such that } \left.\gamma(0)=z_{1}, \gamma(T)=z_{2}\right\} .
\end{gathered}
$$

By the assumption $(\mathrm{H}), d\left(z_{1}, z_{2}\right)<\infty$ for any $z_{1}, z_{2} \in \mathbb{R}^{2}$, and hence it is a metric. To prove this, we will need only to prove that we can connect each pair of points $z_{1}=\left(x_{1}, y_{1}\right)$ and $z_{2}=\left(x_{2}, y_{2}\right)$ by means of a sub-unit curve. Arguing as in $[\mathrm{FL}]$ and $[\mathrm{F}]$, it is easy to see that we can reduce ourselves to the case $x_{1}=x_{2}$ and $\lambda\left(x_{1}\right)=0$. But in that case we note that, by hypothesis $(\mathrm{H})$, the function $s \rightarrow \lambda\left(x_{1}+s\right)$ cannot vanish identically on $(0, t)$ for any $t>0$. Thus, it is enough to move away from $z_{1}$ along the segment $s \rightarrow\left(x_{1}+s, y_{1}\right)$ (which is a sub-unit curve), until we reach a point $\left(\bar{x}, y_{1}\right)$ such that $\lambda(\bar{x})>0$, and then we can 'climb along a vertical' segment up to the point $\left(\bar{x}, y_{2}\right)$ because $s \rightarrow\left(\bar{x}, y_{1}+s\right)$ is also a sub-unit curve. Finally, by repeating backward the previous 'horizontal' segment at the level $y=y_{2}$, we can achieve the proof.

Let us now introduce a function which will play a key role in the description of the metric balls relatives to $d$.

If $z=(x, y) \in \mathbb{R}^{2}$ and $r>0$, put

$$
F(z, r)=F(x, r)=\int_{x}^{x+r} \lambda(s) d s
$$

We shall see later (Theorem 2.3) that $r$ and $F(x, r)$ are respectively the sizes of a metric ball in the directions of the coordinate axis.

In what follows we will say that a constant $c \geq 0$ is a geometric constant if it depends on the constant $c_{1}$ of Hypothesis $(\mathrm{H})$ and on $\sup \lambda$. To avoid cumbersome notation, at many points we will denote by the same letter $c$ different geometric constants. 
We have:

Proposition 2.2 ([FGuW1, Proposition 2.5]). Suppose hypothesis (H) holds. Then for any point $z_{0}=\left(x_{0}, y_{0}\right) \in \mathbb{R}^{2}$ there exist a neighborhood $\mathcal{U}$ of $z_{0}$ and a geometric constant $\gamma \geq 0$ such that

(i) $F(z, \theta t) \geq c \theta^{1+\gamma} F(z, t), 0<\theta<1$;

(ii) $F^{-1}(z, \theta t) \leq c \theta^{1 /(1+\gamma)} F(z, t), 0<\theta<1$;

(iii) $F(z, \theta t) \leq c \theta F(z, t), 0<\theta<1$;

(iv) if $d\left(z_{1}, z_{2}\right)<$ ct, then $F\left(z_{1}, t\right) \sim F\left(z_{2}, t\right)$,

for $0<t<t_{0}$ and $z \in \mathcal{U}$, where $c$ is a geometric constant.

In particular, the following crucial inequality follows from (i):

$$
\int_{0}^{t} \lambda(x+s \xi) d s \geq c t^{1+\gamma}
$$

for any $z=(x, y) \in \mathcal{U}, \xi \in S_{0}=\left\{\xi \in \mathbb{R}:\left|\xi-\xi_{0}\right| \leq \delta\right\} \subset[-1,1] \backslash\{0\}$ and $t \in\left(0, t_{0}\right)$.

We observe that, because of Proposition 2.2 (ii), the following doubling property holds:

$$
F(z, r) \leq F(z, 2 r) \leq c F(z, r)
$$

for any $z \in \mathcal{U}$, and $r<r_{0}, r_{0}$ and $c$ being geometric constants.

We can now combine Proposition 2.2 above with the characterization of the $d$ balls given in $[\mathrm{F}]$, Theorem 2.3. The following theorem contains the description of the geometry given by $d$.

Theorem 2.3. Let the assumption $(H)$ be satisfied. Then:

(i) $d\left(z_{1}, z_{2}\right)<\infty$ for any $z_{1}, z_{2} \in \mathbb{R}^{2}$, and hence $d$ is a metric.

(ii) If we denote by $B(z, r)$ the d-ball centered at $z$ and of radius $r$ (i.e. $B(z, r)=$ $\left.\left\{z^{\prime} \in \mathbb{R}^{2} ; d\left(z, z^{\prime}\right)<r\right\}\right)$, then there exist two geometric constants $t_{1}>0$ and $b>1$ such that, for any $z_{0} \in \mathbb{R}^{2}$ and $t \in\left(0, t_{1}\right)$, we have

$$
Q\left(z_{0}, t / b\right) \subseteq B\left(z_{0}, t\right) \subseteq Q\left(z_{0}, b t\right),
$$

where, for any $r>0$,

$$
Q\left(z_{0}, r\right)=\left\{z=(x, y) \in \mathbb{R}^{2}:\left|x-x_{0}\right|<r \text { and }\left|y-y_{0}\right|<F\left(z_{0}, r\right)\right\} .
$$

(iii) There exist two geometric constants $A>0$ and $r_{0}>0$ such that

$$
|B(z, 2 r)| \leq A|B(z, r)|
$$

for any $z \in \mathcal{U}$ and $r \in\left(0, r_{0}\right)$, i.e. the metric space $\left(\mathbb{R}^{2}, d\right)$ is a space of homogeneous type with respect to Lebesgue measure.

(iv) If $\theta>0$, then

$$
c_{1}(\theta)|B(z, r)| \leq|B(z, \theta r)| \leq c_{2}(\theta)|B(z, r)|
$$

for any $z \in \mathcal{U}$ and $r<r_{0}$ and for some suitable constants $c_{1}(\theta)$ and $c_{2}(\theta)$ which are geometric constants, except for the dependence on $\theta$.

Throughout this paper, we will denote by $\nabla_{\lambda}=\left(\partial_{1}, \lambda \partial_{2}\right)$ the degenerate gradient associated with the operator $\mathcal{L}$, and we will put

$$
\left|\nabla_{\lambda} u\right|^{2}=\left|\partial_{1} u\right|^{2}+\lambda^{2}(x)\left|\partial_{2} u\right|^{2}
$$

Moreover, we will denote by $H_{\lambda}^{1}(\Omega)$ the degenerate Sobolev space associated with $\nabla_{\lambda}$, i.e. the set of $u \in L^{2}(\Omega)$ such that

$$
\partial_{1} u \in L^{2}(\Omega), \quad \lambda \partial_{2} u \in L^{2}(\Omega),
$$


endowed with the natural norm

$$
\|u\|_{H_{\lambda}^{1}(\Omega)}^{2}=\|u\|_{L^{2}(\Omega)}^{2}+\left\|\partial_{1} u\right\|_{L^{2}(\Omega)}^{2}+\left\|\lambda \partial_{2} u\right\|_{L^{2}(\Omega)}^{2}
$$

We note that a Meyers-Serrin type theorem holds for these spaces, i.e.

$$
\mathbf{C}^{\infty}(\Omega) \cap H_{\lambda}^{1}(\Omega) \text { is dense in } H_{\lambda}^{1}(\Omega)
$$

(see $[\mathrm{Fr}],[\mathrm{FSSC}]$ and $[\mathrm{GN}]$ ). Therefore, it will be natural to denote by $\stackrel{\circ}{H}_{\lambda}^{1}(\Omega)$ the closure of $C_{0}^{\infty}(\Omega)$ in $H_{\lambda}^{1}(\Omega)$.

Hypothesis $(\mathrm{H})$ implies suitable forms of classical Sobolev-Poincaré inequalities, where, as we pointed out in the Introduction, the constant $\gamma$ in Proposition 2.2 plays the role of a dimension: see for instance $[\mathrm{F}]$ and [FGuW1]. However, what we need here is only a simple form of this inequality, which states that the $L^{2}$-norm of a compactly supported function in $\Omega$ can be controlled by the $L^{2}$-norm in $\Omega$ of its degenerate gradients, which is therefore equivalent to the norm in $H_{\lambda}^{1}(\Omega)$ (see, e.g., [F], Theorem 4.7).

Theorem 2.4. Suppose Hypothesis (H) holds; then there exists a geometric constant $c>0$ such that

$$
\int_{\Omega}|u|^{2} d z \leq c \int_{\Omega}\left|\nabla_{\lambda} u\right|^{2} d z
$$

for all $u \in \stackrel{\circ}{H}{ }_{\lambda}^{1}(\Omega)$.

Note that Theorem 2.4 implies that, if $u \in \stackrel{\circ}{H}_{\lambda}^{1}(\Omega)$, then

$$
\|u\|_{L^{2}(\Omega)} \leq c\left\|\nabla_{\lambda} u\right\|_{L^{2}(\Omega)},
$$

so that the quadratic form

$$
\mathcal{A}(u, u)=\int_{\Omega}\left|\nabla_{\lambda} u\right|^{2} d z
$$

associated with the operator $\mathcal{L}$ is coercive on $\stackrel{\circ}{H}_{\lambda}^{1}(\Omega)$.

We can now state the main result concerning the Dirichlet problem for $\mathcal{L}$ in $\Omega$.

Theorem 2.5. Let $f_{0}, f=\left(f_{1}, f_{2}\right)$ be such that $f_{0},|f| \in L^{2}(\Omega)$. Then there exists a unique $u \in \stackrel{\circ}{H}_{\lambda}^{1}(\Omega)$ solution of the Dirichlet problem

$$
(P) \quad\left\{\begin{array}{l}
\mathcal{L} u=-\operatorname{div}_{\lambda} f+f_{0} \quad \text { in } \Omega, \\
u=0 \quad \text { on } \Gamma
\end{array}\right.
$$

where $\operatorname{div}_{\lambda} f=\partial_{1} f_{1}+\lambda \partial_{2} f_{2}$, in the sense that

$$
\mathcal{A}(u, \varphi)=\int_{\Omega}\left\{\partial_{1} u \partial_{1} \varphi+\lambda^{2} \partial_{2} u \partial_{2} \varphi\right\} d z=\int_{\Omega}\left\{f_{1} \partial_{1} \varphi+f_{2} \partial_{2} \varphi+f_{0} \varphi\right\} d z=L_{f}(\varphi)
$$

for any $\varphi \in C_{0}^{\infty}(\Omega)$.

The proof follows straightforwardly by standard arguments from the LaxMilgram theorem because of our Poincaré inequality (Theorem 2.4).

Arguing as in [F] and in [FS], Theorems 5.11 and 6.4 respectively, we can prove the following result.

Theorem 2.6. If $f_{0},|f| \in L^{p}$ for $p>p_{0}=p_{0}(\lambda)$, then the solution $u$ of $(P)$ is Hölder continuous in $\bar{\Omega}$. 


\section{Finite element method}

Let us start by constructing a triangulation of $\Omega$ which fits the geometry associated with the operator. Note that the parameter $n$ which will be considered from now on has nothing to do with the dimensionality of the space, which is fixed and equal to 2 .

Theorem 3.1. For any $n>0$ there exists a finite decomposition $\mathcal{T}_{n}$ of the domain

$$
\bar{\Omega}=\bigcup_{K \in \mathcal{T}_{n}} K
$$

where

(i) each $K$ is a compact triangle with nonempty interior $\operatorname{Int}(K)$;

(ii) $\operatorname{Int}\left(K_{1}\right) \cap \operatorname{Int}\left(K_{2}\right)=\emptyset$ for distinct $K_{1}, K_{2} \in \mathcal{T}_{n}$;

(iii) if $F=K_{1} \cap K_{2} \neq \emptyset$, where $K_{1}$ and $K_{2}$ are distinct elements of $\mathcal{T}_{n}$, then $F$ must be a side for both $K_{1}$ and $K_{2}$ or a vertex for both $K_{1}$ and $K_{2}$ (this means that no vertex of one triangle lies on the edge of another triangle);

(iv) for any $K \in \mathcal{T}_{n}$ there exist $\bar{z}_{K}, \overline{\bar{z}}_{K} \in \mathbb{R}^{2}$ and $\bar{r}_{K}, \overline{\bar{r}}_{K}>0$ such that

$$
B\left(\bar{z}_{K}, \bar{r}_{K}\right) \subseteq K \subseteq B\left(\overline{\bar{z}}_{K}, \overline{\bar{r}}_{K}\right), \quad 0<c \leq \frac{\bar{r}_{K}}{\overline{\bar{r}}_{K}} \leq C,
$$

$c$ and $C$ being geometric constants;

(v) $\sup _{K} \bar{r}_{K} \leq$ const $n^{-1 /(1+\gamma)}$, where $\gamma$ is the constant appearing in Proposition 2.2.

Proof. Let us start by constructing the vertices of our triangulation in the set $\bar{\Omega}^{+}$ where $x \geq 0, y \geq 0$. By reflection across the axes we will obtain all the vertices of the triangulation.

First, let us choose $\alpha>0$ such that $\alpha \int_{0}^{1} \lambda(s) d s=1$. Without loss of generality, we can assume that the constant $c_{1}$ in Hypothesis $(\mathrm{H})$ is such that $\alpha c_{1}<1$, and let $\delta_{0}, \delta_{1}, \ldots, \delta_{n}$ be chosen so that

$$
\delta_{0}=0, \quad \alpha \int_{\delta_{j}}^{\delta_{j+1}} \lambda(s) d s=\frac{1}{n} \quad \text { for } \quad j=0,1, \ldots, n-1 .
$$

This choice is always possible by putting $\Lambda(t)=\int_{0}^{t} \lambda(s) d s$ (which is strictly increasing by Hypothesis $(\mathrm{H}))$ and

$$
\delta_{j}=\Lambda^{-1}\left(\frac{j}{\alpha n}\right), \quad j=1, \ldots, n .
$$

Then we can consider the triangulation of $\bar{\Omega}^{+}$given by the family of nodes

$$
\left(\delta_{j}, \frac{k}{n}\right), \quad j=0, \ldots, n ; \quad k=0, \ldots, n .
$$

Note that the nodes

$$
\left(\delta_{n}, \frac{k}{n}\right), \quad k=0, \ldots, n
$$

and

$$
\left(\delta_{j}, \frac{n}{n}\right), \quad j=0, \ldots, n,
$$

belong to $\Gamma$. From the above construction it is clear that $N \sim n^{2}$, where $N$ is the number of nodes in the triangulation. 
It is easy to see that the triangulation associated with this family of nodes satisfies (i)-(iii).

Let us prove (iv). To this end, let $K$ be a triangle of $\mathcal{T}_{n}$. Its vertices will be of the following forms: either

$$
\left(\delta_{j}, \frac{k}{n}\right), \quad\left(\delta_{j+1}, \frac{k}{n}\right), \quad\left(\delta_{j+1}, \frac{k \pm 1}{n}\right), \quad j=0, \ldots, n-1, \quad k=0, \ldots, n-1,
$$

or

$$
\left(\delta_{j}, \frac{k}{n}\right), \quad\left(\delta_{j+1}, \frac{k}{n}\right), \quad\left(\delta_{j}, \frac{k \pm 1}{n}\right), \quad j=0, \ldots, n-1, \quad k=0, \ldots, n-1 .
$$

For simplicity, let us consider only the case with $K$ given by the vertices

$$
P_{1}=\left(\delta_{j}, \frac{k}{n}\right), \quad P_{2}=\left(\delta_{j+1}, \frac{k}{n}\right), \quad P_{3}=\left(\delta_{j}, \frac{k+1}{n}\right) .
$$

Set $\overline{\bar{z}}_{K}=\left(\delta_{j}, \frac{k}{n}\right)$ and $\tilde{r}_{K}=\max \left\{\frac{\alpha}{c_{1}}, 1\right\} \cdot\left(\delta_{j+1}-\delta_{j}\right)$, where $c_{1}$ is the constant of Hypothesis $(\mathrm{H})$. We have

$$
\begin{aligned}
F\left(\overline{\bar{z}}_{K}, \tilde{r}_{K}\right) & =\int_{\delta_{j}}^{\delta_{j}+\tilde{r}_{K}} \lambda(s) d s \geq c_{1} \tilde{r}_{K} \max _{\left[\delta_{j}, \delta_{j}+\tilde{r}_{K}\right]} \lambda \\
& \geq \alpha\left(\delta_{j+1}-\delta_{j}\right) \max _{\left[\delta_{j}, \delta_{j+1}\right]} \lambda \\
& \geq \alpha \int_{\delta_{j}}^{\delta_{j+1}} \lambda(s) d s=\frac{1}{n}=\frac{k+1}{n}-\frac{k}{n},
\end{aligned}
$$

so that:

$$
K \subseteq Q\left(\overline{\bar{z}}_{K}, \tilde{r}_{K}\right) \subseteq B\left(\overline{\bar{z}}_{K}, b \tilde{r}_{K}\right),
$$

and hence the second inclusion in (iv) is proved with $\overline{\bar{r}}_{K}=b \tilde{r}_{K}$. To prove the first inclusion, set

$$
\bar{z}_{K}=\left(\delta_{j}+\theta\left(\delta_{j+1}-\delta_{j}\right), \frac{k+\theta}{n}\right), \quad \tilde{r}_{K}=\varepsilon\left(\delta_{j+1}-\delta_{j}\right),
$$

where $\theta, \varepsilon \in(0,1)$ are fixed constants such that $\theta+\varepsilon<1 /\left(1+\frac{1}{c_{1} \alpha}\right), \quad \varepsilon<c_{1} \alpha \theta$.

Let us prove that $Q\left(\bar{z}_{K}, \tilde{r}_{K}\right) \subset K$, so that, by Theorem 2.3 , we can choose $\bar{r}_{K}=\tilde{r}_{K} / b$. To this end, it will be enough to show that:

(a) the vertex $\left(\delta_{j}+\theta\left(\delta_{j+1}-\delta_{j}\right)+\varepsilon\left(\delta_{j+1}-\delta_{j}\right), \frac{k+\theta}{n}+F\left(\bar{z}_{K}, \tilde{r}_{K}\right)\right)$ lies below the line $z_{2}=\frac{1}{n\left(\delta_{j+1}-\delta_{j}\right)}\left\{-z_{1}+k\left(\delta_{j+1}-\delta_{j}\right)+\delta_{j+1}\right\}=\psi\left(z_{1}\right)$ (which connects $P_{2}$ and $\left.P_{3}\right)$,

(b) the vertex $\left(\delta_{j}+\theta\left(\delta_{j+1}-\delta_{j}\right)-\varepsilon\left(\delta_{j+1}-\delta_{j}\right), \frac{k+\theta}{n}-F\left(\bar{z}_{K}, \tilde{r}_{K}\right)\right)$ lies above the line $z_{2}=\frac{k}{n}$ (which connects $P_{1}$ and $P_{2}$ ), and

(c) the vertex $\left(\delta_{j}+\theta\left(\delta_{j+1}-\delta_{j}\right)-\varepsilon\left(\delta_{j+1}-\delta_{j}\right), \frac{k+\theta}{n}-F\left(\bar{z}_{K}, \tilde{r}_{K}\right)\right)$ lies on the right of the line $z_{1}=\delta_{j}$ (which connects $P_{1}$ and $P_{3}$ ). 
Indeed:

(a)

$$
\begin{aligned}
\frac{k+\theta}{n} & +F\left(\bar{z}_{K}, \tilde{r}_{K}\right)=\frac{k+\theta}{n}+\int_{\delta_{j}+\theta\left(\delta_{j+1}-\delta_{j}\right)}^{\delta_{j}+\theta\left(\delta_{j+1}-\delta_{j}\right)+\varepsilon\left(\delta_{j+1}-\delta_{j}\right)} \lambda(s) d s \\
& \leq \frac{k+\theta}{n}+\varepsilon\left(\delta_{j+1}-\delta_{j}\right) \max _{\left[\delta_{j}+\theta\left(\delta_{j+1}-\delta_{j}\right), \delta_{j}+(\theta+\varepsilon)\left(\delta_{j+1}-\delta_{j}\right)\right]} \lambda \\
& \leq \frac{k+\theta}{n}+\varepsilon\left(\delta_{j+1}-\delta_{j}\right) \max _{\left[\delta_{j}, \delta_{j+1}\right]} \lambda \\
& \left(\text { since } \delta_{j+1}-\delta_{j}>0 \text { and } \theta+\varepsilon<1\right) \\
& \leq \frac{k+\theta}{n}+\frac{\varepsilon}{c_{1}} \int_{\delta_{j}}^{\delta_{j+1}} \lambda(s) d s \\
& (\text { by Hypothesis }(\mathrm{H})) \\
& =\frac{k+\theta}{n}+\frac{\varepsilon}{\alpha c_{1} n}<\frac{1}{n}(1-\theta-\varepsilon+k) \\
& \left(\text { since } 2<1+\frac{1}{c_{1} \alpha}\right) \\
& =\psi\left(\delta_{j}+\theta\left(\delta_{j+1}-\delta_{j}\right)+\varepsilon\left(\delta_{j+1}-\delta_{j}\right)\right) .
\end{aligned}
$$

(b)

$$
\begin{aligned}
\frac{k+\theta}{n}-F\left(\bar{z}_{K}, \tilde{r}_{K}\right) & \geq \frac{k+\theta}{n}-\frac{\varepsilon}{\alpha c_{1} n} \\
& (\text { arguing as above) } \\
& =\frac{k}{n}+\frac{1}{n}\left(\theta-\frac{\varepsilon}{\alpha c_{1}}\right)>\frac{k}{n} .
\end{aligned}
$$

(c) $\delta_{j}+\theta\left(\delta_{j+1}-\delta_{j}\right)-\varepsilon\left(\delta_{j+1}-\delta_{j}\right)>\delta_{j}$, since $\varepsilon<c_{1} \alpha \theta<\theta$.

To achieve the proof of (iv), we note that both $\bar{r}_{K}$ and $\overline{\bar{r}}_{K}$ are given by a geometric constant times $\delta_{j+1}-\delta_{j}$, so that assertion (iv) is completely proved.

On the other hand, by (3.1) and (2.2),

$$
\frac{1}{\alpha n}=\int_{\delta_{j}}^{\delta_{j+1}} \lambda(s) d s=\xi_{0} \int_{0}^{\left(\delta_{j+1}-\delta_{j}\right) / \xi_{0}} \lambda\left(\delta_{j}+s \xi_{0}\right) d s \geq c\left(\xi_{0}\right)\left(\delta_{j+1}-\delta_{j}\right)^{1+\gamma},
$$

and then $(\mathrm{v})$ is proved.

We can proceed now in a standard way by defining a finite dimensional space $V_{n}$ in the following way: Let $P_{1}, \ldots, P_{N}, N=N(n)$ be the nodes of $\mathcal{T}_{n}$ which belong to $\operatorname{Int}(\Omega)$. We consider the set $\Phi_{n}$ of all continuous piecewise linear functions $\varphi_{j}, j=1, \ldots, N$, such that

$$
\varphi_{j} \equiv 0 \text { on } \Gamma \quad \text { and } \quad \varphi_{j}\left(P_{i}\right)=\delta_{i j}, \quad i=1, \ldots, N,
$$

and we denote by $V_{n}$ the linear space generated by $\Phi_{n}$.

Lemma 3.2. $V_{n} \subset \stackrel{\circ}{H}_{\lambda}^{1}(\Omega)$ for $n \geq 1$

Proof. It is enough to note that $V_{n} \subset \stackrel{\circ}{H}^{1}(\Omega) \subset \stackrel{\circ}{H}_{\lambda}^{1}(\Omega)$. 
A function $v_{n} \in V_{n}$ now has the representation

$$
v_{n}(z)=\sum_{i=1}^{N} v_{n}\left(P_{i}\right) \varphi_{i}(z),
$$

and our Dirichlet problem can be approximated by the following one.

Find $u_{n} \in V_{n}$ such that

$$
\left(P_{n}\right) \quad A\left(u_{n}, v_{n}\right)=L_{f}\left(v_{n}\right) \quad \forall v_{n} \in V_{n} .
$$

As in the elliptic theory, the above problem can be solved by solving an $N \times N$ linear system of equations whose stiffness matrix has elements given by

$$
\left(a\left(\varphi_{i}, \varphi_{j}\right)\right)_{i, j=1, \ldots, N} \cdot
$$

We will see in Section 5 a discussion of a numerical solution of this problem.

Again as in the classical theory, we can define an interpolation operator $\Pi_{n}: \mathbf{C}^{0}(\bar{\Omega}) \rightarrow V_{n}$ as follows:

$$
\Pi_{n}(v)=\sum_{i=1}^{N} v\left(P_{i}\right) \varphi_{i} .
$$

\section{Error estimate}

Suppose now that $f=\left(f_{1}, f_{2}\right)$ belongs to $L^{p}(\Omega)$ with $p>p_{0}$ as in Theorem 2.6, so that the solution $u$ of the Dirichlet problem (2.10) is continuous on $\bar{\Omega}$.

We will follow the classical Galerkin approximation scheme (see [QV], 6.2.1). This technique provides us with an error estimate giving the rate of convergence of the approximate solutions $u_{n}$ to $u$ in the norm of the space of weak solutions $H_{\lambda}^{1}(\Omega)$. It must be noticed that this error estimate is optimal, as will be clear from the numerical results reported in Section 5. As in the usual elliptic case, the error estimates rely on $L^{2}$ estimates of the second derivatives of $u$; however, because of the lack of ellipticity when $x=0$, we cannot expect usual $H^{2}$ estimates to hold, but, in the spirit of our approach, if we denote by $X_{1}, X_{2}$ the vector fields $\partial_{1}$ and $\lambda(x) \partial_{2}$ respectively, our second order degenerate Sobolev space $H_{\lambda}^{2}(\Omega)$ will consist of these $u \in H_{\lambda}^{1}(\Omega)$ such that each monomial $X_{i} X_{j} u$ belongs to $L^{2}(\Omega)$ for $i, j=1,2$, endowed with its natural norm.

Unfortunately, the corresponding estimates up to the boundary seem rather hard to obtain. Nevertheless, if we restrict ourselves to a diagonal operator of the form

$$
\mathcal{L}_{0}=-\partial_{1}^{2}-\lambda^{2}(x) \partial_{2}^{2},
$$

where $\lambda$ is a $\mathbf{C}^{0,1}$ function satisfying Hypothesis $(\mathrm{H})$, these boundary estimates can be deduced from analogous interior estimates.

For instance, if $\lambda^{2}=\mu^{2}$, where $\mu$ is a smooth function such that $\mu^{(m)}(0) \neq 0$ for some $m>0$, then these a priori interior estimates for second order 'derivatives' hold as a particular case of a deep result of Rotschild and Stein ([RS]). Note that if $\lambda$ has such a form, then Hypothesis (H) is automatically satisfied (see below). For instance, the prototype Grushin operator corresponding to $\lambda(x)=|x|^{\gamma}$ satisfies this assumption when $\gamma \in \mathbb{N}$ (note that the choice of the symbol $\gamma$ for the exponent is not casual here, since it is consistent with (2.2)).

Let us start with the following general result that does not rely on any particular structure of $\mathcal{L}$. 
Theorem 4.1. Suppose $\lambda$ is a $\mathbf{C}^{0,1}$ function satisfying Hypothesis (H) such that $\lambda^{2} \in \mathbf{C}^{1,1}$, its zeros are isolated and belong to $\operatorname{Int}(\Omega)$, and that $\left(\lambda^{2}\right)^{\prime \prime} \geq 0$ in a neighborhood of these zeros. If $f \in L^{\infty}(\Omega)$, then there exists a unique $u \in$ $\stackrel{\circ}{H}_{\lambda}^{1}(\Omega) \cap \mathbf{C}^{0, \sigma}(\bar{\Omega})$ for some $\sigma \in(0,1)$ such that

$$
\mathcal{L} u=f \quad \text { in } \Omega \text {. }
$$

If in addition $X_{i} X_{j} u \in L^{2}(\Omega)$ for $i, j=1,2$ (where $X_{1}=\partial_{1}, X_{2}=\lambda(x) \partial_{2}$ ), then the Galerkin approximations $u_{n} \in V_{n}$ defined by (3.7) converge to $u \in \stackrel{\circ}{H^{1}}(\Omega)$ as $n \rightarrow \infty$ and the following error estimate holds:

$$
\left\|u-u_{n}\right\|_{H_{\lambda}^{1}(\Omega)} \leq B n^{-1 /(1+\gamma)}
$$

where $B$ depends on $\|f\|_{L^{2}(\Omega)}$ and on $\left\|X_{i} X_{j} u\right\|_{L^{2}(\Omega)}, i, j=1,2$.

Remark. Suppose in addition we know that for any $f \in L^{2}(\Omega)$ we have $u \in H_{\lambda}^{2}(\Omega) \cap$ $\stackrel{\circ}{H}_{\lambda}^{1}(\Omega)$. This implies that the map $u \rightarrow \mathcal{L} u$ is a bijection from $H_{\lambda}^{2}(\Omega) \cap \stackrel{\circ}{H}_{\lambda}^{1}(\Omega)$ onto $L^{2}(\Omega)$, and hence, by the closed graph theorem, the following a priori estimate holds:

$$
\sum_{i, j=1}^{2}\left\|X_{i} X_{j} u\right\|_{L^{2}(\Omega)} \leq C\|f\|_{L^{2}(\Omega)} .
$$

If such an estimate holds, then the error estimate can be written in the form

$$
\left\|u-u_{n}\right\|_{H_{\lambda}^{1}(\Omega)} \leq B n^{-1 /(1+\gamma)}\|f\|_{L^{2}(\Omega)}
$$

where $B$ is a geometric constant.

Proof of Theorem 4.1. First of all we notice that $f \in \bigcap_{p \geq 1} L^{p}(\Omega)$, and hence $u \in$ $\stackrel{\circ}{H}_{\lambda}^{1}(\Omega) \cap \mathbf{C}^{0, \sigma}(\bar{\Omega})$ for some $\sigma \in(0,1)$ by Theorem 2.6 .

Without loss of generality, we may assume that $\lambda(0)=0$ and $\lambda(x)>0$ if $x \neq 0$. Moreover, we can assume that $\lambda\left(x^{\prime}\right) \geq \lambda\left(x^{\prime \prime}\right)$ for $0 \leq x^{\prime \prime} \leq x^{\prime}$ and $\lambda\left(x^{\prime}\right) \leq \lambda\left(x^{\prime \prime}\right)$ for $x^{\prime \prime} \leq x^{\prime} \leq 0$. As in $[\mathrm{QV}]$, Theorem 5.2.1, there exist $B_{1}, B_{2}>0$, depending only on $\nu$ and the constant $c$ of (2.9), such that

$$
\begin{gathered}
\left\|u_{n}\right\|_{H_{\lambda}^{1}(\Omega)} \leq B_{1}\|f\|_{L^{2}(\Omega)}, \\
\left\|u_{n}-u\right\|_{H_{\lambda}^{1}(\Omega)} \leq B_{2} \inf _{v_{n} \in V_{n}}\left\|u-v_{n}\right\|_{H_{\lambda}^{1}(\Omega)} .
\end{gathered}
$$

On the other hand,

$$
\inf _{v_{n} \in V_{n}}\left\|u-v_{n}\right\|_{H_{\lambda}^{1}(\Omega)} \leq\left\|u-\Pi_{n}(u)\right\|_{H_{\lambda}^{1}(\Omega)}
$$

since $\Pi_{n}(u)$ is well defined because of the continuity of $u$ and it belongs to $V_{n}$ because of Lemma 3.2. By combining (4.2) and (4.3) it will follow that $u_{n} \rightarrow u$ in $H_{\lambda}^{1}(\Omega)$ once we have proved that $\left\|u-\Pi_{n}(u)\right\|_{H_{\lambda}^{1}(\Omega)} \rightarrow 0$ as $n \rightarrow \infty$.

Now

$$
\begin{aligned}
\left\|u-\Pi_{n}(u)\right\|_{H_{\lambda}^{1}(\Omega)} & \leq c\left\{\left\|u-\Pi_{n}(u)\right\|_{L^{2}(\Omega)}+\left\|\nabla_{\lambda}\left(u-\Pi_{n}(u)\right)\right\|_{L^{2}(\Omega)}\right\} \\
& =c \sum_{K \in \mathcal{T}_{n}}\left\{\left\|u-\Pi_{n}(u)\right\|_{L^{2}(K)}+\left\|\nabla_{\lambda}\left(u-\Pi_{n}(u)\right)\right\|_{L^{2}(K)}\right\} .
\end{aligned}
$$


We have:

Lemma 4.2. There exists a geometric constant $B_{3}>0$ such that, if we put $X_{1}=$ $\partial_{1}, X_{2}=\lambda \partial_{2}$, then, if $K \in \mathcal{T}_{n}$,

$$
\begin{aligned}
& \left\|u-\Pi_{n}(u)\right\|_{L^{2}(K)}+\left\|\nabla_{\lambda}\left(u-\Pi_{n}(u)\right)\right\|_{L^{2}(K)} \\
& \quad \leq B_{3}\left\{\left\|X_{1}^{2} u\right\|_{L^{2}(K)}+\left\|X_{2}^{2} u\right\|_{L^{2}(K)}+\left\|X_{2} X_{1} u\right\|_{L^{2}(K)}\right\} n^{-1 /(1+\gamma)} .
\end{aligned}
$$

Note that $\operatorname{Int}(K)$ intersects neither $\Gamma$ nor the degeneration line $x=0$, so that $u \in \mathbf{C}_{l o c}^{2, \alpha}(\operatorname{Int} K)$, by classical Schauder estimates.

Proof. First, let us estimate

$$
\int_{K}\left|\nabla_{\lambda}\left(u-\Pi_{n}(u)\right)\right|^{2} d x d y=\int_{K}\left|\nabla_{\lambda}\left(u-\sum_{i=1}^{3} u\left(Q_{i}\right) \varphi_{i}\right)\right|^{2} d x d y=I_{2},
$$

where $Q_{1}, Q_{2}, Q_{3}$ are the vertices of $K$.

Suppose for instance that $K$ has vertices $\left(\delta_{j}, \frac{k}{n}\right),\left(\delta_{j+1}, \frac{k}{n}\right),\left(\delta_{j}, \frac{k+1}{n}\right)$, and put

$$
\left\{\begin{array}{l}
x=\delta_{j}+\left(\delta_{j+1}-\delta_{j}\right) x^{\prime} \\
y=\frac{k}{n}+\frac{1}{n} y^{\prime}
\end{array}\right.
$$

The above change of variables maps $K$ onto the base triangle of vertices $(0,0),(1,0)$, $(0,1)$, and its Jacobian determinant is $2|K|$, so that, if we denote by $\tilde{v}$ any function $v$ written in the new variables $x^{\prime}, y^{\prime}$, we get

$$
\int_{K}\left|v-\Pi_{n}(v)\right|^{2} d x d y=2|K| \int_{\tilde{K}}\left|\tilde{v}-\sum_{i=1}^{3} \tilde{v}\left(\tilde{Q}_{i}\right) \tilde{\varphi}_{i}\right|^{2} d x^{\prime} d y^{\prime}
$$

Note now that

$$
\partial_{x^{\prime}} \tilde{v}=\left(\delta_{j+1}-\delta_{j}\right) \widetilde{\partial_{x} v}, \quad \partial_{y^{\prime}} \tilde{v}=\frac{1}{n} \widetilde{\partial_{y} v} .
$$

Then

$$
\begin{aligned}
\int_{K}\left|\partial_{x}\left(u-\sum_{i=1}^{3} u\left(Q_{i}\right) \varphi_{i}\right)\right|^{2} d x d y & =2|K| \int_{\tilde{K}}\left|\widetilde{\partial_{x}(\ldots)}\right|^{2} d x^{\prime} d y^{\prime} \\
& =\frac{2|K|}{\left(\delta_{j+1}-\delta_{j}\right)^{2}} \int_{\tilde{K}}\left|\partial_{x^{\prime}} \widetilde{(\ldots)}\right|^{2} d x^{\prime} d y^{\prime} \\
& =\frac{1}{n\left(\delta_{j+1}-\delta_{j}\right)} \int_{\tilde{K}}\left|\partial_{x^{\prime}} \widetilde{(\ldots)}\right|^{2} d x^{\prime} d y^{\prime} .
\end{aligned}
$$

Analogously

$$
\begin{aligned}
\int_{K} \mid \lambda(x) \partial_{y}(u & \left.-\sum_{i=1}^{3} u\left(Q_{i}\right) \varphi_{i}\right)\left.\right|^{2} d x d y \\
& =n\left(\delta_{j+1}-\delta_{j}\right) \int_{\tilde{K}} \lambda^{2}\left(\delta_{j}+\left(\delta_{j+1}-\delta_{j}\right) x^{\prime}\right)\left|\partial_{y^{\prime}} \widetilde{(\ldots)}\right|^{2} d x^{\prime} d y^{\prime} \\
& \leq n\left(\delta_{j+1}-\delta_{j}\right) \cdot\left(\max _{\left[\delta_{j}, \delta_{j+1}\right]} \lambda\right)^{2} \int_{\tilde{K}}\left|\partial_{y^{\prime}} \widetilde{(\ldots)}\right|^{2} d x^{\prime} d y^{\prime} \\
& \leq \frac{1}{c_{1}^{2}} \frac{n}{\left(\delta_{j+1}-\delta_{j}\right)}\left(\int_{\delta_{j}}^{\delta_{j+1}} \lambda(t) d t\right)^{2} \int_{\tilde{K}}\left|\partial_{y^{\prime}} \widetilde{(\ldots)}\right|^{2} d x^{\prime} d y^{\prime} \\
& =c \frac{1}{n\left(\delta_{j+1}-\delta_{j}\right)} \int_{\tilde{K}}\left|\partial_{y^{\prime}} \widetilde{\ldots}\right|^{2} d x^{\prime} d y^{\prime}, \quad \text { by }(3.1),
\end{aligned}
$$


so that

$$
I_{2} \leq c \frac{1}{n\left(\delta_{j+1}-\delta_{j}\right)}\left|\tilde{u}-\sum_{i=1}^{3} \tilde{u}\left(\tilde{Q}_{i}\right) \tilde{\varphi}_{i}\right|_{H^{1}(\tilde{K})}^{2},
$$

where $\tilde{Q}_{1}, \tilde{Q}_{2}, \tilde{Q}_{3}$ are vertices of $\tilde{K}$, and $|\cdot|_{H^{k}(\tilde{K})}$ denotes the seminorm given by the sum of the $L^{2}$-norms of the highest derivatives. We can now apply the following classical estimate:

$$
\left|\tilde{u}-\sum_{i=1}^{3} \tilde{u}\left(\tilde{Q}_{i}\right) \tilde{\varphi}_{i}\right|_{H^{1}(\tilde{K})}^{2} \leq c|\tilde{u}|_{H^{2}(\tilde{K})}^{2}
$$

(see, for instance $[\mathrm{QV}]$, Theorem 3.4.1), and we get

$$
\begin{aligned}
& I_{2} \leq c \frac{1}{n\left(\delta_{j+1}-\delta_{j}\right)}\left\{\left\|\partial_{x^{\prime}}^{2} \tilde{u}\right\|_{L^{2}(\tilde{K})}^{2}+\left\|\partial_{y^{\prime}}^{2} \tilde{u}\right\|_{L^{2}(\tilde{K})}^{2}+\left\|\partial_{y^{\prime}} \partial_{x^{\prime}} \tilde{u}\right\|_{L^{2}(\tilde{K})}^{2}\right\} \\
&=c\left\{\widetilde{\left(\delta_{j+1}-\delta_{j}\right)^{3}}\left\|\widetilde{\partial_{x}^{2} u \|_{L^{2}(\tilde{K})}^{2}}+\frac{1}{n}\right\| \widetilde{n^{5}\left(\delta_{j+1}-\delta_{j}\right)}\left\|\widetilde{\partial_{y}^{2} u}\right\|_{L^{2}(\tilde{K})}^{2}\right. \\
&\left.+\frac{\left(\delta_{j+1}-\delta_{j}\right)}{n^{3}}\left\|\widetilde{\partial_{y} \partial_{x} u}\right\|_{L^{2}(\tilde{K})}^{2}\right\} \\
&= c\left\{\left(\delta_{j+1}-\delta_{j}\right)^{2}\left\|\partial_{x}^{2} u\right\|_{L^{2}(K)}^{2}+\frac{1}{n^{4}}\left\|\partial_{y}^{2} u\right\|_{L^{2}(K)}^{2}+\frac{1}{n^{2}}\left\|\partial_{y} \partial_{x} u\right\|_{L^{2}(K)}^{2}\right\} \\
&=\left\{J_{1}+J_{2}+J_{3}\right\} .
\end{aligned}
$$

First of all, we note that $J_{1}$ can be written as follows:

$$
J_{1}=\left(\delta_{j+1}-\delta_{j}\right)^{2}\left\|X_{1}^{2} u\right\|_{L^{2}(K)}^{2}
$$

the next step will consist of proving that

$$
J_{2} \leq c\left(\delta_{j+1}-\delta_{j}\right)^{2}\left\|X_{2}^{2} u\right\|_{L^{2}(K)}^{2} \quad \text { and } \quad J_{3} \leq c\left(\delta_{j+1}-\delta_{j}\right)^{2}\left\|X_{2} X_{1} u\right\|_{L^{2}(K)}^{2} .
$$

To this end, we observe that, if $x \in K$, then, by the monotonicity of $\lambda$,

$$
\lambda(x) \geq \lambda\left(\delta_{j}\right) \geq \frac{1}{\delta_{j}-\delta_{j-1}} \int_{\delta_{j-1}}^{\delta_{j}} \lambda(t) d t=\frac{1}{n\left(\delta_{j}-\delta_{j-1}\right)},
$$

so that (4.13) will follow by proving that

$$
\delta_{j}-\delta_{j-1} \leq c\left(\delta_{j+1}-\delta_{j}\right)
$$

for all $j=0,1, \ldots, n-1$.

On the other hand, it is easy to see that

$$
d\left(\left(\delta_{j-1}, k / n\right),\left(\delta_{j}, k / n\right)\right) \leq \delta_{j}-\delta_{j-1},
$$

so that, by Proposition 2.2 (iv), we have

$$
\begin{aligned}
F\left(\left(\delta_{j}, k / n\right),\right. & \left.\delta_{j}-\delta_{j-1}\right) \leq c F\left(\left(\delta_{j-1}, k / n\right), \delta_{j}-\delta_{j-1}\right) \\
& =c \int_{\delta_{j-1}}^{\delta_{j}} \lambda(t) d t=\frac{c}{\alpha n}=c \int_{\delta_{j}}^{\delta_{j+1}} \lambda(t) d t \\
& =c F\left(\left(\delta_{j}, k / n\right), \delta_{j+1}-\delta_{j}\right) \leq F\left(\left(\delta_{j}, k / n\right), c^{\prime}\left(\delta_{j+1}-\delta_{j}\right)\right),
\end{aligned}
$$

by Proposition 2.2 (iii), and hence (4.14) follows by the monotonicity of the function $t \rightarrow F\left(\left(\delta_{j}, k / n\right), t\right)$. 
Thus

$$
\begin{aligned}
I_{2} & \leq c\left(\delta_{j+1}-\delta_{j}\right)^{2}\left\{\left\|X_{1}^{2} u\right\|_{L^{2}(K)}^{2}+\left\|X_{2}^{2} u\right\|_{L^{2}(K)}^{2}+\left\|X_{2} X_{1} u\right\|_{L^{2}(K)}^{2}\right\} \\
& \leq n^{-2 /(1+\gamma)}\left\{\left\|X_{1}^{2} u\right\|_{L^{2}(K)}^{2}+\left\|X_{2}^{2} u\right\|_{L^{2}(K)}^{2}+\left\|X_{2} X_{1} u\right\|_{L^{2}(K)}^{2}\right\},
\end{aligned}
$$

by Theorem 3.1 (iv) and (v). Finally, the term

$$
I_{1}=\int_{K}\left|u-\Pi_{n}(u)\right|^{2} d x d y
$$

can be handled in the same way.

Combining (4.4) and (4.5), we complete the proof of Theorem 4.1.

Theorem 4.3. Let $\lambda$ be as in Theorem 4.1, and let the following interior a priori estimate hold: if $v \in H_{\lambda, l o c}^{1}\left(\mathbb{R}^{2}\right)$ is such that $\mathcal{L}_{0} v=g \in L^{2}(\Omega)$, then for any $\psi \in C_{0}^{\infty}\left(\mathbb{R}^{2}\right)$

$$
\sum_{i, j=1,2}\left\|X_{i} X_{j}(\psi v)\right\|_{L^{2}(\Omega)} \leq C_{\psi}\|g\|_{L^{2}(\Omega)}
$$

Then the following error estimate for the Galerkin approximations of the solution $u \in \stackrel{\circ}{H}_{\lambda}^{1}(\Omega)$ of $\mathcal{L}_{0} u=f \in L^{\infty}(\Omega)$ holds:

$$
\left\|u-u_{n}\right\|_{H_{\lambda}^{1}(\Omega)} \leq B n^{-1 /(1+\gamma)}\|f\|_{L^{2}(\Omega)},
$$

where $B$ is a geometric constant.

Proof. By Theorem 4.1 and the subsequent remark, we have only to prove that $X_{i} X_{j} u \in L^{2}(\Omega)$ for $i, j=1,2$. Consider the following covering $\left\{F_{j}\right\}$ for $\Omega$ : $\left.F_{1}=\right]-\infty,-1 / 4\left[\times \mathbb{R}, F_{2}=\right] 1 / 4,+\infty\left[\times \mathbb{R}, F_{3}=\right]-1 / 3,1 / 3[\times]-3 / 4,3 / 4\left[, F_{4}=e_{2}\right.$ $+]-1 / 3,1 / 3[\times]-1 / 3,1 / 3\left[, F_{5}=-e_{2}+\right]-1 / 3,1 / 3[\times]-1 / 3,1 / 3\left[\right.$, where $e_{2}=(0,1)$. Let moreover $\left\{\psi_{j}, j=1,2,3,4,5\right\}$ be a partition of unity subordinate to the covering $\left\{F_{j}\right\}$, with $\psi_{j} \in \mathbf{C}_{0}^{\infty}\left(\mathbb{R}^{2}\right), 0 \leq \psi_{j} \leq 1$, supp $\psi_{j} \subset F_{j}$. We have

$$
\sum_{i, j}\left\|X_{i} X_{j} u\right\|_{L^{2}(\Omega)} \leq \sum_{k} \sum_{i, j}\left\|X_{i} X_{j}\left(\psi_{k} u\right)\right\|_{L^{2}\left(F_{k}\right)} .
$$

Consider now $k=1,2$ : these cases can be easily reduced to usual elliptic estimates. Indeed, in $F_{1}$ and $F_{2}$ the $H_{\lambda}^{2}$ norm is equivalent to the usual Sobolev norm, for $\lambda$ is bounded away from zero on these regions. On the other hand, for the same reason, the operator $\mathcal{L}_{0}$ is elliptic on $F_{1}$ and $F_{2}$, and then it satisfies standard $H^{2}$ a priori estimates by a well known regularity result on planar angular regions due to P. Grisvard ([G]). Thus, if we take into account that

$$
\mathcal{L}_{0}\left(\psi_{k} u\right)=\psi_{k} f-2\left\langle\nabla_{\lambda} \psi_{k}, \nabla_{\lambda} u\right\rangle-u \mathcal{L}_{0} \psi_{k}=g_{k},
$$

and that $\left\|g_{k}\right\|_{L^{2}(\Omega)} \leq c\|f\|_{L^{2}(\Omega)}$, we get for $k=1,2$

$$
\begin{aligned}
\sum_{i, j}\left\|X_{i} X_{j}\left(\psi_{k} u\right)\right\|_{L^{2}\left(F_{k}\right)} & \leq c\left\|\psi_{k} u\right\|_{H^{2}(\Omega)} \leq c\left\|\mathcal{L}_{0}\left(\psi_{k} u\right)\right\|_{L^{2}(\Omega)}+\left\|\psi_{k} u\right\|_{L^{2}(\Omega)} \\
& \leq c\|f\|_{L^{2}(\Omega)} .
\end{aligned}
$$

Thus we can restrict ourselves to considering, for instance, the region $F_{4}$. We set $\left.F_{4}=Q, Q_{-}=e_{2}+\right]-1 / 3,1 / 3[\times]-1 / 3,0\left[\right.$ and $\left.Q_{+}=e_{2}+\right]-1 / 3,1 / 3[\times] 0,1 / 3[$. We can assume that $\psi_{4}=\psi$ satisfies $\psi \equiv 1$ on $B\left(e_{2}, 1 / 4\right)$. 
As above, $u \psi \in \stackrel{\circ}{H_{\lambda}^{1}}(\Omega)$ and $\mathcal{L}_{0}(u \psi)=g \in L^{2}(\Omega)$, with $\|g\|_{L^{2}(\Omega)} \leq c\|f\|_{L^{2}(\Omega)}$. We now define

$$
G_{1}(x, y)=\left\{\begin{array}{lll}
g & \text { in } & Q_{-} \\
0 & \text { in } & Q_{+}
\end{array}\right.
$$

and we denote by $v_{1} \in \stackrel{\circ}{H}_{\lambda}^{1}(Q)$ the solution of the problem

$$
\left(P_{1}\right)\left\{\begin{array}{l}
\mathcal{L}_{0} v_{1}=G_{1} \quad \text { in } \quad Q, \\
v_{1}=0 \quad \text { on } \quad \partial Q .
\end{array}\right.
$$

Let $G_{2}(x, y)=G_{1}(x, 2-y)$ and let $v_{2} \in \stackrel{\circ}{H}_{\lambda}^{1}(Q)$ be the solution of the problem

$$
\left(P_{2}\right) \quad\left\{\begin{array}{l}
\mathcal{L}_{0} v_{2}=G_{2} \quad \text { in } \quad Q, \\
v_{2}=0 \quad \text { on } \quad \partial Q .
\end{array}\right.
$$

We have:

(1) $v_{1}, v_{2} \in C^{0, \alpha}(\bar{Q})$;

(2) $v_{2}(x, y)=v_{1}(x, 2-y)$.

The first property holds as above, arguing as in [FS], whereas the second property follows from the uniqueness of the solution of $\left(P_{2}\right)$, if we prove that $v_{1}(x, 2-y)$ solves $\left(P_{2}\right)$. To this end, let $\varphi \in C_{0}^{\infty}(Q)$; then

$$
\begin{aligned}
\int_{Q}\left[\left(v_{1}\right)_{x}(x,\right. & \left.2-y) \varphi_{x}(x, y)-\lambda^{2}(x)\left(v_{1}\right)_{2-y}(x, 2-y) \varphi_{y}(x, y)\right] d x d y \\
& =\int_{Q}\left[\left(v_{1}\right)_{x}(x, \eta) \varphi_{x}(x, 2-\eta)-\lambda^{2}(x)\left(v_{1}\right)_{\eta}(x, \eta) \varphi_{2-\eta}(x, 2-\eta)\right] d x d \eta \\
& =\int_{Q}\left[\left(v_{1}\right)_{x}(x, \eta) \psi_{x}(x, \eta)+\lambda^{2}(x)\left(v_{1}\right)_{\eta}(x, \eta) \psi_{\eta}(x, \eta)\right] d x d \eta \\
& (\text { where } \psi(x, \eta)=\varphi(x, 2-\eta)) \\
& =\int_{Q} G_{1}(x, \eta) \psi(x, \eta) d x d \eta=\int_{Q} G_{1}(x, \eta) \varphi(x, 2-\eta) d x d \eta \\
& =\int_{Q} G_{1}(x, 2-y) \varphi(x, y) d x d y \\
& =\int_{Q} G_{2}(x, y) \varphi(x, y) d x d y
\end{aligned}
$$

Now we put $v=\left.\left(v_{1}-v_{2}\right)\right|_{\bar{Q}_{-}}$, so that $v(x, y)=\left.\left(v_{1}(x, y)-v_{1}(x, 2-y)\right)\right|_{\bar{Q}_{-}}, v \in$ $C^{0, \alpha}\left(\bar{Q}_{-}\right)$and $v \equiv 0$ on $\partial Q_{-}$. Moreover, $v \in H_{\lambda}^{1}\left(Q_{-}\right)$. We assume we have proved that $v \in \stackrel{\circ}{H}_{\lambda}^{1}\left(Q_{-}\right)$, and we verify that $\mathcal{L}_{0} v=g$ in $Q_{-}$. Let $\varphi \in C_{0}^{\infty}\left(Q_{-}\right)$; then

$$
\begin{aligned}
\int_{Q_{-}} & \left(v_{x} \varphi_{x}+\lambda^{2} v_{y} \varphi_{y}\right) d x d y \\
& =\int_{Q_{-}}\left[\left(v_{1}\right)_{x} \varphi_{x}+\lambda^{2}\left(v_{1}\right)_{y} \varphi_{y}\right] d x d y-\int_{Q_{-}}\left[\left(v_{2}\right)_{x} \varphi_{x}+\lambda^{2}\left(v_{2}\right)_{y} \varphi_{y}\right] d x d y \\
& =\int_{Q_{-}}\left(G_{1}-G_{2}\right) \varphi d x d y=\int_{Q_{-}} g \varphi d x d y,
\end{aligned}
$$

since $G_{1} \equiv g$ in $Q_{-}$and $G_{2} \equiv 0$ in $Q_{-}$. Hence by uniqueness $v=u \psi$. 
We prove now that if $v \in H_{\lambda}^{1}\left(Q_{-}\right) \cap C^{0, \alpha}\left(\bar{Q}_{-}\right)$and $v \equiv 0$ on $\partial Q_{-}$, then $v \in \stackrel{\circ}{H}_{\lambda}^{1}\left(Q_{-}\right)$. To this end we consider the covering for $Q_{-}$given by $E_{1}=Q_{-} \cap$ (]$-\infty,-1 / 4[\times \mathbb{R}), E_{2}=Q_{-} \cap(] 1 / 4,+\infty[\times \mathbb{R}), E_{3}=Q_{-} \cap(\mathbb{R} \times] 1-2 \delta,+\infty[), E_{4}=$ $Q_{-} \cap(\mathbb{R} \times] 1-\delta,-\infty[)$, and let $\left\{\varphi_{i}, i=1,2,3,4,\right\}$ be a partition of unity subordinate to this covering. It will be enough to prove that $v \varphi_{i} \in \stackrel{\circ}{H}_{\lambda}^{1}\left(Q_{-}\right)$for $i=1,2,3,4$. This is clear for $v \varphi_{1}$ and $v \varphi_{2}$, since $v \varphi_{1}, v \varphi_{2} \in H^{1}\left(Q_{-}\right)$and so $v \in \stackrel{\circ}{H}^{1}\left(Q_{-}\right) \subseteq \stackrel{\circ}{H}_{\lambda}^{1}\left(Q_{-}\right)$by the usual results on Sobolev spaces. We now prove that $v \varphi_{3}=\tilde{v} \in \stackrel{\circ}{H}_{\lambda}^{1}\left(Q_{-}\right) ; v \varphi_{4}$ can be treated analogously. To prove that $\tilde{v} \in \stackrel{\circ}{H}_{\lambda}^{1}\left(Q_{-}\right)$ we will prove that $\tilde{v}$ can be approximated by functions $\tilde{v}_{n} \in H_{\lambda}^{1}\left(Q_{-}\right)$such that supp $\tilde{v}_{n} \subset Q_{-}$. Indeed, since the usual convolutions with Friedrich's mollifiers do converge in $H_{\lambda}^{1}\left(Q_{-}\right)$([FSSC], [GN]), each $\tilde{v}_{n}$ can be approximated in $H_{\lambda}^{1}\left(Q_{-}\right)$by functions in $C_{0}^{\infty}\left(Q_{-}\right)$, and hence the statement follows.

Let $\psi_{n}:[0,+\infty) \rightarrow[0:+\infty)$ be a smooth function such that $\psi_{n} \equiv 1$ on $[0,1-1 / n], \psi_{n} \equiv 0$ on $[1-1 / 2 n,+\infty), 0 \leq \psi_{n} \leq 1,\left|\psi_{n}^{\prime}\right| \leq 3 n$ : we set $\tilde{v}_{n}=\psi_{n} \tilde{v}$. It follows that $\operatorname{supp} \tilde{v}_{n} \subset Q_{-}$and $\left\|\tilde{v}_{n}-\tilde{v}\right\|_{L^{2}\left(Q_{-}\right)}=\left\|\tilde{v}\left(\psi_{n}-1\right)\right\|_{L^{2}\left(Q_{-}\right)}$. Now $\tilde{v}\left(\psi_{n}-1\right) \rightarrow 0$ a.e. and $\left|\tilde{v}\left(\psi_{n}-1\right)\right| \leq|\tilde{v}|$, and hence the norm tends to zero. Therefore $\tilde{v}_{n} \rightarrow \tilde{v}$ in $L^{2}\left(Q_{-}\right)$. Analogously $\partial_{x} \tilde{v}_{n} \rightarrow \partial_{x} \tilde{v}$ in $L^{2}\left(Q_{-}\right)$. Assume we have proved that $\left\|\lambda \partial_{y} \tilde{v}_{n}\right\|_{L^{2}\left(Q_{-}\right)} \leq C$. It follows from the reflexivity of $H_{\lambda}^{1}\left(Q_{-}\right)$ that $\left(\tilde{v}_{n}\right)_{n \in \mathbb{N}}$ converges weakly in $H_{\lambda}^{1}\left(Q_{-}\right)$, and then $\tilde{v}_{n} \rightarrow \tilde{v}$ weakly in $H_{\lambda}^{1}\left(Q_{-}\right)$ since $\tilde{v} \in H_{\lambda}^{1}\left(Q_{-}\right)$and $\tilde{v}_{n} \rightarrow \tilde{v}$ in $L^{2}\left(Q_{-}\right)$. Hence, by Mazur's theorem, $\tilde{v}$ is the limit in $H_{\lambda}^{1}\left(Q_{-}\right)$of a sequence of finite convex combinations of $\left\{\tilde{v}_{k}, k \in \mathbb{N}\right\}$ which are still functions supported in $Q_{-}$, and we are done.

Let us prove now that $\left\|\lambda \partial_{y} \tilde{v}_{n}\right\|_{L^{2}\left(Q_{-}\right)}$is bounded. We notice that

$$
\frac{\partial \tilde{v}}{\partial y} \in L^{2}\left(Q_{-} \cap\{|x| \geq \varepsilon\}\right)
$$

for every $\varepsilon>0$, and hence the function $y \rightarrow \frac{\partial \tilde{v}}{\partial y}(x, y)$ is in $L_{l o c}^{1}$ for almost every $x$. Therefore, using the property $\tilde{v}(x, 1) \equiv 0$, we have

$$
|\tilde{v}(x, y)| \leq \int_{y}^{1}\left|\frac{\partial \tilde{v}}{\partial y}(x, t)\right| d t \leq \sqrt{1-y}\left(\int_{y}^{1}\left|\frac{\partial \tilde{v}}{\partial y}\right|^{2} d t\right)^{1 / 2}
$$

for $(x, y) \in Q_{-}$. We then have

$$
\left\|\lambda \partial_{y} \tilde{v}_{n}\right\|_{L^{2}\left(Q_{-}\right)} \leq\left\|\lambda \psi_{n}\left(\partial_{y} \tilde{v}\right)\right\|_{L^{2}\left(Q_{-}\right)}+\left\|\lambda \tilde{v}\left(\partial_{y} \psi_{n}\right)\right\|_{L^{2}\left(Q_{-}\right)} .
$$

The first term tends to $\left\|\lambda \partial_{y} \tilde{v}\right\|_{L^{2}\left(Q_{-}\right)}$as $n \rightarrow \infty$, as above. Concerning the second one, we have

$$
\begin{aligned}
\left\|\lambda \tilde{v}\left(\partial_{y} \psi_{n}\right)\right\|_{L^{2}\left(Q_{-}\right)}^{2} & \leq 9 n^{2} \int_{-1 / 3}^{1 / 3} d x \lambda^{2}(x) \int_{1-1 / n}^{1} d y \tilde{v}^{2}(x, y) \\
& \leq 9 n^{2} \int_{-1 / 3}^{1 / 3} d x \lambda^{2}(x) \int_{1-1 / n}^{1} d y(1-y) \int_{2 / 3}^{1}\left|\frac{\partial \tilde{v}}{\partial y}(x, t)\right|^{2} d t \\
& =9 n^{2} \int_{Q_{-}} d x d t\left|\lambda \frac{\partial \tilde{v}}{\partial y}(x, t)\right|^{2} \int_{1-1 / n}^{1} d y(1-y) \\
& =\frac{9}{2}\left\|\lambda \frac{\partial \tilde{v}}{\partial y}\right\|_{L^{2}\left(Q_{-}\right)}^{2},
\end{aligned}
$$

and the statement is proved. 
Now results on interior regularity for problems $\left(P_{1}\right)$ and $\left(P_{2}\right)$ guarantee that $X_{1}^{2} v_{i}, X_{1} X_{2} v_{i}, X_{2} X_{1} v_{i}, X_{2}^{2} v_{i} \in L^{2}\left(B\left(e_{2}, 1 / 4\right)\right)$, and hence it follows that $X_{1}^{2} u$, $X_{1} X_{2} u, X_{2} X_{1} u, X_{2}^{2} u \in L^{2}(\Omega)$.

Corollary 4.4. Let $\lambda$ be such that $\lambda=|\mu|$, where $\mu$ is a smooth function such that

$$
\mu(0)=\mu^{\prime}(0)=\cdots=\mu^{(m-1)}(0)=0
$$

and $\mu^{(m)}(0) \neq 0$ for some $m \geq 1$. Then the conclusion of Theorem 4.3 holds.

Proof. The two vector fields $Y_{1}=\partial_{1}, Y_{2}=\mu \partial_{2}$ satisfy Hörmander's rank condition since the rank of the Lie algebra generated by $Y_{1}$ and $Y_{2}$ equals 2 at each point of $\mathbb{R}^{2}$. Thus the interior a priori estimate of Theorem 4.3 follows from Theorem 16 (d) in [RS]. Thus we have only to show that Hypothesis $(H)$ is satisfied, since the remaining assumptions of Theorem 4.1 follow straightforwardly. On the other hand, Hypothesis (H) follows by Proposition 5 in [FW], where it is shown in particular that, because of the Hörmander condition, the function $\mu$ belongs to $R H_{\infty}$, i.e. its average on any interval is equivalent to its $L^{1}$ norm on the same interval, and so we are done.

\section{Algorithm And numerical Results}

In this section we will describe some numerical tests of our previous results; as we pointed out in the Introduction, the number $\gamma+2$ plays the role of a dimension, which can be very large if the operator is strongly degenerate. Because of this, to test the trend of our estimates we have to work with a mesh containing a large number of points, and, for this reason, we have to choose our implementation rather carefully. The algorithm we used to perform our numerical integrations is MGGHAT, a unified multilevel adaptive refinement method, in which a unified approach to the combined processes of adaptive refinement and multigrid solution has been very conveniently implemented. A detailed technical description of the method can be found in [M1], [M2].

In our case the refinement cannot be obviously performed by bisecting pairs of triangles, since we want to reproduce the geometry associated with the differential operator considered. The hierarchical basis scheme can nevertheless be applied, since also for the geometries considered in this paper it is possible to implement divisions (which reproduce the geometry required) of a pair of triangles, corresponding to the addition of a new basis function having support for the pair of triangles divided, and leaving the existing basis functions unchanged; in fact we modified the part of the program producing the mesh generation, according to our geometry.

Indeed, from (3.2) it can be seen that at each level of refinement all the old nodes are maintained, and some new ones are added. This is exactly the principle on which the hierarchical basis approach is based. The hierarchical basis coincides with the usual nodal basis at the first level of refinement. As refinement proceeds, with each division one or more new nodes are added, and for each node a new basis function is defined so that it has the value 1 at the new node and 0 at all other nodes, but the existing basis functions remain unchanged. The choice of hierarchical basis leads to a representation of $\Pi_{n}(u)$ which differs from (3.8), and then to a different stiffness matrix: the algorithmical gain is described in [BDY] and $[\mathrm{M} 2]$. 
As an example, we choose

$$
\lambda=\sqrt{\beta} m|x|^{m-1} \text { for } \beta>0 \text { and } m>1,
$$

so that it is easy to check that (2.2) holds with $\gamma=m-1$. Put

$$
R=\beta|x|^{2 m}+y^{2} \quad \text { and } \quad u=R^{1 / m},
$$

and, finally,

$$
v=\left(1-x^{2}\right)\left(1-y^{2}\right) u .
$$

A direct calculation shows that $v \in \stackrel{\circ}{H}_{\lambda}^{1}(\Omega)$, and that $f=\mathcal{L}_{0} v \in L^{\infty}(\Omega)$; on the other hand, if $m>2.5$, then $v$ does not belong to the usual Sobolev space $\stackrel{\circ}{H}^{1}(\Omega)$. This fact is not surprising, since the function $u$ is equivalent to the square of the distance $d$ of the point $(x, y)$ from the origin (see [FL]), so that it reflects in a rather subtle way the properties of the model operator $\mathcal{L}_{0}$ and it is strictly connected with the Sobolev space $\stackrel{\circ}{H}_{\lambda}^{1}(\Omega)$.

We can now evaluate the discretization error in the energy norm

$$
\left\|\partial_{x}\left(v-v_{n}\right)\right\|_{L^{2}(\Omega)}+\left\|\lambda \partial_{y}\left(v-v_{n}\right)\right\|_{L^{2}(\Omega)},
$$

which not only is the norm naturally associated with the operator, but it also is the only 'reasonable' norm, since in general the $H^{1}$-norm of $v$ is infinite. By Theorem 2.4, the energy norm is equivalent to the norm in $\stackrel{\circ}{H}_{\lambda}^{1}(\Omega)$.

In the following pictures we plotted these errors in a log-log scale as a function of $N$, the number of the nodes of our triangulation, which we recall is proportional to $n^{2}$ (we call the triangulation corresponding to the geometry naturally associated to the operator the natural triangulation, see Figure 3), and we compared it with the errors we obtain (for the same number of nodes) by using an adaptive version of our triangulation, a uniform triangulation and an adaptive uniform triangulation. The graphs in Figure 1 correspond to $\beta=128$ and $m=4(\gamma=3)$ and $m=6$ $(\gamma=5)$, respectively. The choice of a large $\beta$ has been suggested by the need of amplifying the behaviors we are interested in studying.

Finally, we evaluated the trend of the error estimate, reported in Table 1, by comparing it with the theoretical estimate (always in the same logarithmic scale) in the cases $m=2(\gamma=1)$ and $m=3(\gamma=2)$. The expected trend for the errors is of the form $N^{b}$, where by our error estimate $b=-0.25$ for $\gamma=1$ and $b=-0.1 \overline{6}$ for $\gamma=2$. The results obtained from the linear fitting of the data, i.e. $b=-0.242 \pm 0.008$ for $\gamma=1$ and $b=-0.17 \pm 0.01$ for $\gamma=2$, shown in Figure 2, are a clear indication of the optimality of the error estimate. 

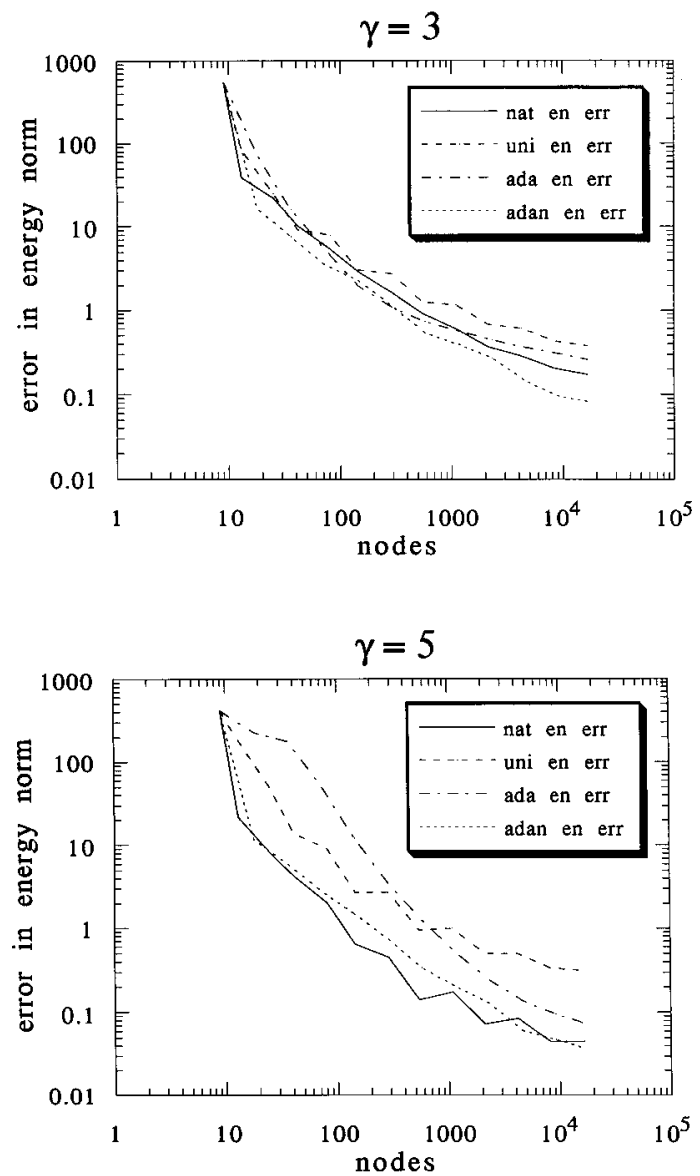

Figure 1. Plot of the discretization error in energy norm, as a function of the number of nodes $N$, obtained with four different triangulation types: natural (i.e. obtained using the geometry naturally associated to the operator), uniform (i.e. obtained using Euclidean geometry), adaptive uniform (i.e. obtained by an adaptive refinement method in the Euclidean geometry) and adaptive natural (i.e. obtained by an adaptive refinement method in the geometry naturally associated to the operator); for $\gamma=3(m=4)$ (Figure 1a, top) and $\gamma=5(m=6)$ (Figure 1b, bottom). 

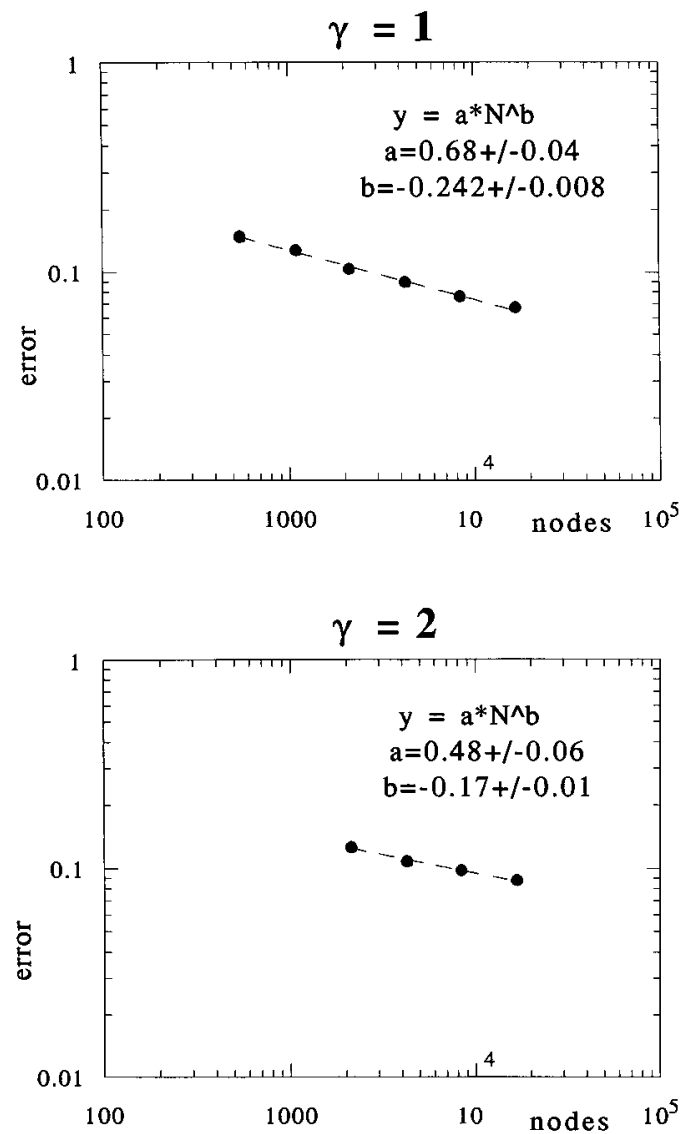

FiguRE 2. Fitting of the discretization error in $H_{\lambda}^{1}$ norm for $\gamma=1(m=2)$ (Figure 2a, top) and $\gamma=2(m=3)$ (Figure $2 \mathrm{~b}$, bottom). The smaller nodes have been discarded to consider only the asymptotic regime. The line superimposed on the data is the result of numerical fit. The data have been fitted with the linear function $f(N)=a \times N^{b}$, where $N$ is the number of nodes used in the triangulation. We obtained the values $b=-0.242 \pm 0.008$ for $\gamma=1$, corresponding to the theoretical prediction $b=-0.25$, and $b=-0.17 \pm 0.01$ for $\gamma=2$, corresponding to the theoretical prediction $b=-0.1 \overline{6}$. The set of data used are also reported in Table 1. 


\section{TABLE 1}

\begin{tabular}{|c|c|c|}
\hline$N$ & $\gamma=1$ & $\gamma=2$ \\
\hline 545 & 0.1483 & \\
\hline 1089 & 0.1279 & \\
\hline 2113 & 0.1039 & 0.1265 \\
\hline 4225 & 0.0895 & 0.1080 \\
\hline 8321 & 0.0763 & 0.0978 \\
\hline 16641 & 0.0674 & 0.0877 \\
\hline
\end{tabular}

gamma $=1$, grid = natural, mxtri $=128$

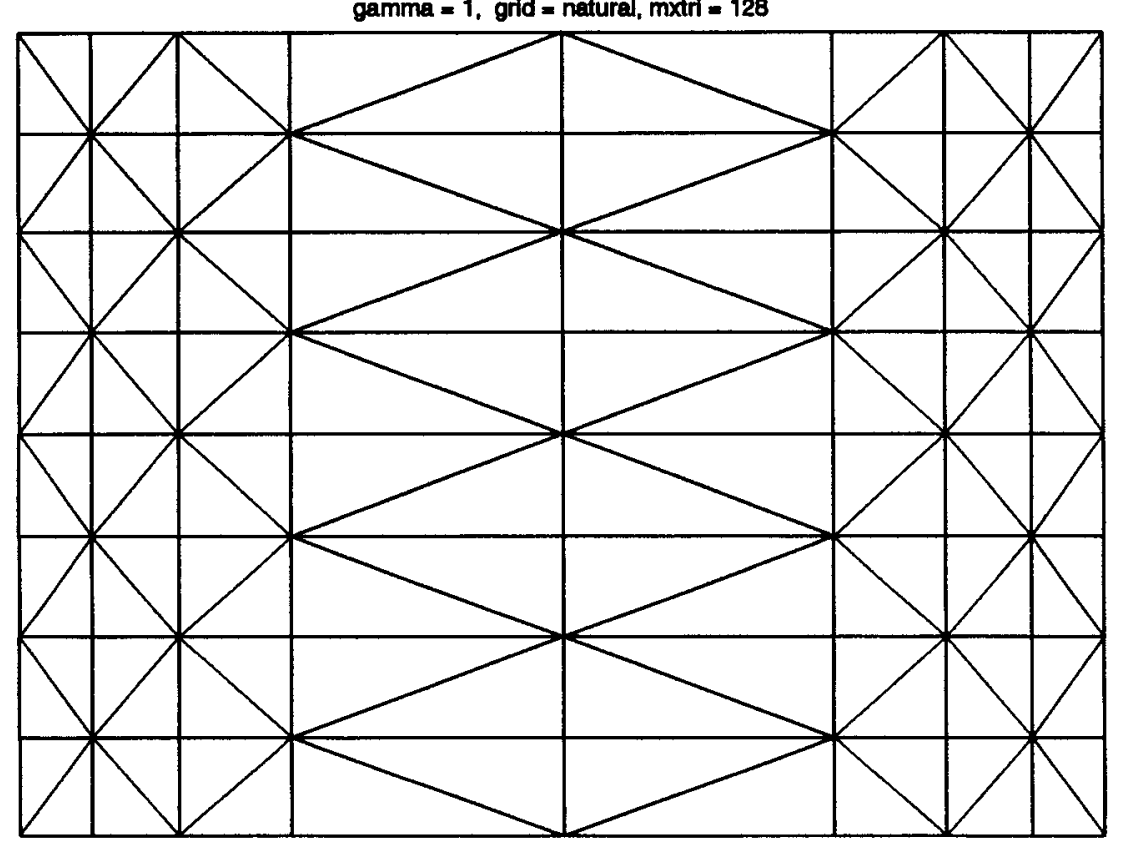

FIGURE 3. Example of a triangulation with 128 triangles obtained with the geometry naturally associate to the operator, with $\gamma=$ $1(m=2)$.

\section{REFERENCES}

[BDY] R.E. Bank, T.F. Dupont and H. Yserentant, The hierarchical basis multigrid method, Num. Math. 52 (1988), 427-458. MR 89b:65247

[CDG1] L. Capogna, D. Danielli and N. Garofalo, The geometric Sobolev embedding for vector fields and the isoperimetric inequality, Comm. in Analysis and Geometry 2 (1994), 203-215. MR 96d:46032

[CDG2] Subelliptic mollifiers and a basic pointwise estimate of Poincaré type, Math. Z. 226 (1997), 147-154. MR 98i:35025

[CF] C. Cancelier and B. Franchi, Subelliptic estimates for a class of degenerate elliptic integro-differential operators, Math. Nachr. 183 (1997), 19-41. MR 98d:35086

[DG] E. De Giorgi, Sulla differenziabilità e l'analiticità degli integrali multipli regolari, Mem. Accad. Sci. Torino Cl. Sci. Fis. Mat. Natur. 3 (1957), 25-43. MR 20:172 
[F] B. Franchi, Weighted Sobolev-Poincaré inequalities and pointwise estimates for a class of degenerate elliptic equations, Trans. Amer. Math. Soc. 327 (1991), 125-158. MR 91m:35095

[Fr] K.O. Friedrichs, The identity of weak and strong estension of differential operators, Trans. Amer. Math. Soc. 55 (1944), 132-151. MR 5:188b

[FGaW] B. Franchi, S. Gallot and R.L. Wheeden, Sobolev and isoperimetric inequalities for degenerate metrics, Math. Ann. 300 (1994), 557-571. MR 96a:46066

[FGuW1] B. Franchi, C. Gutierrez and R.L. Wheeden, Weighted Sobolev-Poincaré inequalities for Grushin type operators, Comm. Partial Differential Equations 19 (1994), 523-604. MR 96h:26019

[FGuW2] B. Franchi, C. Gutierrez and R.L. Wheeden, Two-weight Sobolev-Poincaré inequalities and Harnak inequality for a class of degenerate elliptic operators, Atti Accad. Naz. Lincei, Cl. Sci. Fis. Mat. Natur 5 (9) (1994), 167-175. MR 95i:35115

[FL] B. Franchi and E. Lanconelli, Hölder regularity theorem for a class of linear nonuniformly elliptic operators with measurable coefficients, Ann. Scuola Norm. Sup. Pisa 10 (4) (1983), 523-541. MR 85k:35094

[FLW] B. Franchi, G. Lu and R.L. Wheeden, Representation formulas and weighted Poincaré inequalities for Hörmander vector fields, Ann. Inst. Fourier, Grenoble 45 (1995), 577604. MR 96i: 46037

[FP] C. Fefferman and D.H. Phong, Subelliptic eigenvalue problems, Conference on Harmonic Analysis, Chicago, 1980, (W. Beckner et al., eds.), Wadsworth, 1981, pp. 590-606. MR 86c:35112

[FS] B. Franchi and R. Serapioni, Pointwise estimates for a class of strongly degenerate elliptic operators: a geometrical approach, Ann. Scuola Norm. Sup. Pisa 14 (4) (1987), 527-568. MR 90e:35076

[FSSC] B. Franchi, R. Serapioni and F. Serra Cassano, Champs de vecteurs, théorème d'approximation de Meyers-Serrin et phénomème de Lavrentev pour des fonctionnelles dégénérés, C.R. Acad. Sci. Paris Sér. I Math. 320 (1995), 695-698. MR 95m:46044

[FW] B. Franchi and R.L. Wheeden, Compensation couples and isoperimetric estimates for vector fields, Colloq. Math 74 (1997), 9-27. MR 98g:46042

[G] P. Grisvard, Behaviour of the solutions of an elliptic boundary value problem in a polygonal or polyhedral domain, Numerical Solutions of Partial Differential Equations, III (B. Hubbard, ed.), Academic Press, New York, 1976, pp. 207-274. MR 57:6786

[Gr] M. Gromov, Carnot-Carathéodory spaces seen from within, Sub-Riemannian Geometry, Birkhäuser, 1996, pp. 79-323. CMP 97:04

[GN] N. Garofalo and D.M. Nhieu, Isoperimetric and Sobolev inequalities for Carnot-Carathéodory spaces and the existence of minimal surfaces, Comm. Pure Appl. Math. 49 (1996), 1081-1144. MR 97i:58032

[GT] D. Gilbarg and N.S. Trudinger, Elliptic Partial Differential Equations of Second Order, Springer, Berlin, 1977. MR 57:13109

[J] V. Jurdjevic, Geometric Control Theory, Cambridge Studies in Advanced Mathematics, 1997. MR 98a:93002

[Mo] J. Moser, A new proof of De Giorgi's theorem concerning the regularity problem for elliptic differential equations, Comm. Pure Appl. Math. 13 (1961), 457-468. MR 30:332

[M1] W.F. Mitchell, Unified multilevel adaptive finite element method for elliptic problems, Ph.D. thesis, Report No. UIUCDSC-R-88-1436, Department of Computer Science, University of Illinois, Urbana, IL, 1988.

[M2] W.F. Mitchell, Optimal multilevel iterative methods for adaptive grids, SIAM J. Sci. Stat. Comput. 13 (1992), 146-167. MR 92j:65187

[MP] C. Mogavero and S. Polidoro, A finite difference method for a boundary value problem related to the Kolmogorov equation, Calcolo 32 (1995), 193-206. CMP 98:04

[NSW] A. Nagel, E.M. Stein and S. Wainger, Balls and metrics defined by vector fields I: basic properties, Acta Math. 155 (1985), 103-147. MR 86k:46049

[QV] A. Quarteroni and A. Valli, Numerical Approximation of Partial Differential Equations, Springer Series in Computational Mathematics, Springer, Berlin, 1994. MR 95i:65006

[RS] L.P. Rothschild and E.M.Stein, Hypoelliptic differential operators and nilpotent groups, Acta Math. 137 (1976), 247-320. MR 55:9171 
[X] C.-J. Xu, The Harnack's inequality for second order degenerate elliptic operators, Chinese Ann. Math. Ser A 10 (1989), 359-365, in Chinese. MR 90m:35039

Dipartimento Matematico dell'Università, Piazza di Porta S. Donato, 5, 40127 BoloGNA, ITALY

E-mail address: franchib@dm.unibo.it

Université de Paris-Sud, Mathématiques, BÂt. 425, 91405 Orsay Cedex, France

E-mail address: Maria-Carla.Tesi@math.u-psud.fr 